\title{
CFD ANALYSIS OF DIFFUSER IN A CAR FOR DOWNFORCE GENERATION
}

\author{
Asif Ahmed A ${ }^{1}$, Murtaza M.A ${ }^{2}$ \\ ${ }^{1}$ Research Scholar, Automobile Engineering, Amity University (Lucknow Campus),Uttar Pradesh, India \\ ${ }^{2}$ Professor, ASET, Amity University (Lucknow Campus), Uttar Pradesh, India
}

\begin{abstract}
Many accidents take place on our highways on a daily basis where the driver loses control of his/her vehicle. Highways being the arteries of a nation are used widely by the people to travel and at faster speeds causing many such accidents. While drag reduction is essential on its own, downforce generation plays a pivotal role in improving handling characteristics of the given vehicle. The objective of this paper is to understand how a diffuser works and what advantages it may give to any given vehicle pertaining to its role in altering the drag and lift characteristics of the said vehicle. Computational Fluid Dynamics technique was utilized to determine optimum diffuser angle for maximum downforce that it can create. Ansys 14.0 Fluent was utilized to analyze the drag and lift acting on the vehicle at different diffuser angles at different vehicle speeds.
\end{abstract}

Keywords: Downforce, Diffuser, CFD, Drag, Ansys, Fluent.

\section{INTRODUCTION}

India has world's second largest road network with roads totaling a length of over 3,300,000 kms [1]. And at the same time fatalities due to road accidents jumped from 91,376 in 2004 to 118,239 in 2008 which is more than any other country in the world [2]. Narrowing it down further we come across a startling fact that while the National Highways constitute just $2 \%$ of the total road lengths they carry $40 \%$ of the nation's road traffic and added to that estimates suggest that around $40 \%$ of road fatalities occur at National Highways [1]. This averages to about one fatality per kilometer per year on the National Highway which highlights the dangers of highway driving [3].

Since 2005 UK has been studying crashes and segregating them according to the crash type. It showed that cause of vehicle crash because of any vehicle defect was at a measly $2.8 \%$ whereas due to driver error or reaction it rises to $65.3 \%$ in fatalities. The driver error has been further broken down into different causes among which the most prominent are:

-Loss of control: $34 \%$

-Poor Turn: $12 \%$

- Swerved: $6.2 \%[4]$

All these data suggest that drivers usually struggle to get to grips with the car at higher speeds. This is mostly because of the very large amount of turbulence being created behind the car body and also the effect of lift forces causing the driver to lose traction. Lift forces increase with increase in velocity and drivers then feel like the rear of the car is "floating around" while at cruising speeds. Remedies have been made by using Electronic Stability Control (ESC). While it has been an effective tool to reduce loss of control of the car by the driver, the loss of traction at the rear wheels due to lift forces is still present and it causes drop in confidence of the driver which can be dangerous. This can be improved impressively by incorporating the use of diffusers in car bodies. The simulations have been carried out using Computational Fluid Dynamics (CFD) which is flexible method to study effects of wind flow on a car body. Ansys Fluent was used to conduct the simulations.

\section{OBJECTIVE}

This paper focuses on the CFD analysis of a diffuser in a production car. The vehicle selected for the simulation is Honda City as it is a very popular vehicle and is among the most used vehicles for highway travels. Also availability of its dimensions for CAD design was an advantage.

The vehicle model was created in Solidworks 2014 and then was imported into Ansys Fluent Workbench which is a solver. The target was to simulate the air flow around the vehicle body and obtain the drag and lift coefficients for the body. Then a diffuser was designed and incorporated to the car body and the same simulations were performed for different diffuser angles at different wind velocities in order to create downforce on the vehicle allowing it to handle better at high speeds.

\section{THEORY}

\subsection{Drag and Lift Forces}

When a vehicle is travelling on the road it is subjected to a lot of forces be it mechanical or aerodynamic which acts upon the vehicle from all directions. In case of aerodynamic forces they are split up into various directions as the wind flows around the body of the vehicle. Lift is the component of this aerodynamic force that is perpendicular to the oncoming flow direction. On the other hand the force component which is parallel to the flow direction is termed as drag force. 


\subsection{Computational Fluid Dynamics}

J.D.Anderson Jr. [5] describes CFD as the art of replacing the integrals or the partial derivatives in these equations with discretized algebraic forms, which in turn are solved to obtain numbers for the flow field values at discrete points in time/space. Computers are used to calculate via iteration methodology where every iteration converges the equation to a more accurate solution. The underlying equations that solve the problem are the Reynolds Averaged Navier-Stokes Equations (RANS). Reynolds decomposition is used to separate time averaged and fluctuating quantities of an instantaneous quantity. The time averaged nature of its equations makes it a preferred choice for simulating turbulent flows. Based on the knowledge of properties of turbulent flows, these equations can be used to give time averaged solutions to the Navier-Stokes equations [8]. While track testing is the final assessment in terms of performance and production, it is rarely used for developing new designs. CFD gives a lot of leeway to keep updating our designs and solve it to see which design gives best results and then it is used to validate on track data. Hence CFD is considered as very vital for designing and ground effect aerodynamics is the area where it probably has the greatest area of growth [6].

\subsection{Diffuser}

Diffuser is a device which operates on the Bernoulli's Principle which states that as the velocity of the air increases, its pressure decreases as is represented by the following equation, from point 1 to point 2 on the same streamline. The underbody of the vehicle acts as a venturi to pinch the air hence lowering the pressure and force it to move faster relative to the air above the car. This pressure differential results in increased load (down force) on the tyres [5].

$$
p_{1}+\frac{1}{2} \rho V_{1}^{2}=p_{2}+\frac{1}{2} \rho V_{2}^{2}
$$

Rear diffuser is a shaped section of the underbody of the car and is normally closed on either ends by end-plates so as to prohibit unwanted spreading of the air flow around the rear. As shown in the fig1.

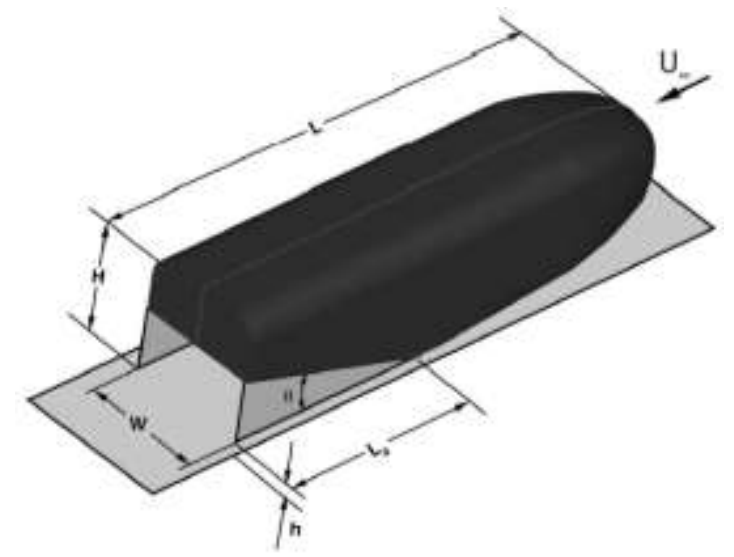

Fig 1.A simple illustration of a rear diffuser on a bluff body to study aerodynamics in ground effect
This configuration of the underbody results in increased downforce thus resulting in enhanced overall mechanical grip. The flow volume between the ground and the body is strongly dependent on the car's attitude relative to the ground [9]. By gradually increasing the cross-sectional area of the diffuser, the air slows down gradually and returns to its free-stream velocity and pressure. As the vehicle sits very close to the ground, (Fig. 2), the lift coefficient is quite high as the flow of air is restricted. But as the ground clearance increases to around $50 \mathrm{~mm}$ it gives maximum downforce and as the clearance increases the lift coefficient reduces due to wide opening of the diffuser mouth which results in vortex generation and also the velocity of the air is quite fast due to larger cross-section leading to lower pressure acting downwards on the body.

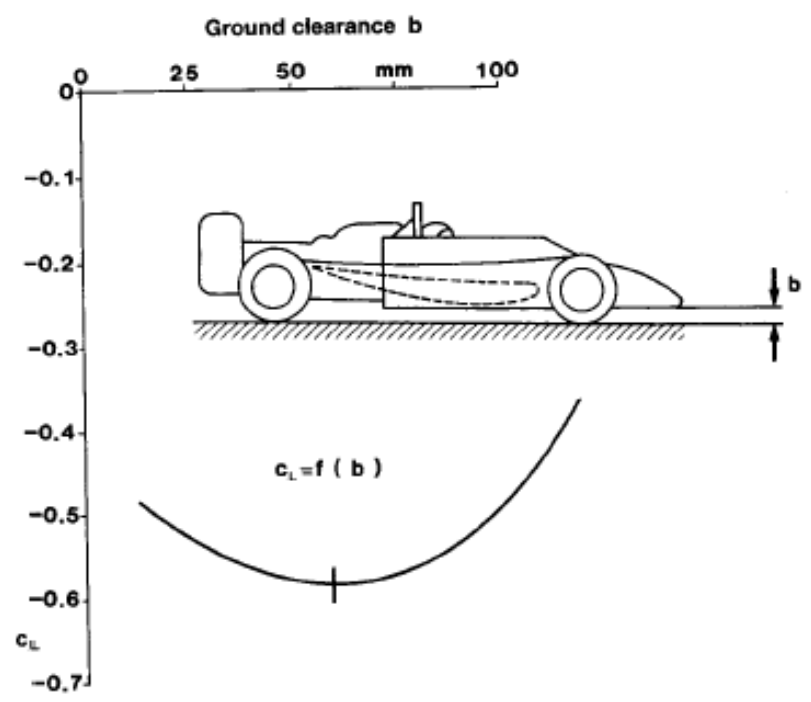

Fig2: Correlation between lift coefficient and ground clearance.

Cooper et al. [10] used CFD was used to simulate airflow through the diffuser. A 3D model with 9.17 and $13.5 \mathrm{deg}$ diffusers were simulated on a symmetric half-model without the side plates. RANS was performed for the simulation and $\mathrm{k}-\varepsilon$ turbulence model was used. It gave satisfactory values for both lift and pressure in case of diffuser set at $9.17 \mathrm{deg}$, whereas the simulation was not as much successful for the $13.5 \mathrm{deg}$ diffuser. They then analytically modeled the same and utilized the earlier results to find out design data for the diffuser that can be deemed acceptable [11]. It resulted in very insightful results in the designing of rear diffusers such as: an optimum area ratio parameter of approximately $(\mathrm{AR}=)$ 1-2 and the length of the diffuser to be more or less of around half the length of the vehicle.

\subsection{ANSYS Solver}

\section{Pressure-Based Coupled Solver}

The pressure-based coupled solver (PBCS) was introduced in 2006 by ANSYS Inc. It reduces the time for the convergence by about five times, as it solves momentum and pressure-based continuity equations in a coupled manner. It does require increased memory requirements but the advantages of using it far outweigh the negatives [12]. 


\section{k-epsilon Model}

$\mathrm{k}$-epsilon $(\mathrm{k}-\varepsilon)$ turbulence model is the most common model used in CFD to simulate turbulent flow conditions. It is a two equation model which employs two transport equations to represent the turbulent properties of the flow.

For turbulent kinetic energy k [13]

$\mathrm{Z}$

$\frac{\partial(\rho k)}{\partial t}+\frac{\partial\left(\rho k u_{i}\right)}{\partial x_{i}}=\frac{\partial}{\partial x_{j}}\left[\frac{\mu_{t}}{\sigma_{k}} \frac{\partial k}{\partial x_{j}}\right]+2 \mu_{t} E_{i j} E_{i j}-\rho \epsilon$

For dissipation $\varepsilon$

$\frac{\partial(\rho \epsilon)}{\partial t}+\frac{\partial\left(\rho \epsilon u_{i}\right)}{\partial x_{i}}=\frac{\partial}{\partial x_{j}}\left[\frac{\mu_{t}}{\sigma_{\epsilon}} \frac{\partial \epsilon}{\partial x_{j}}\right]+C_{1 \epsilon} \frac{\epsilon}{k} 2 \mu_{t} E_{i j} E_{i j}-C_{2 \epsilon} \rho \frac{\epsilon^{2}}{k}$

Where, $\mu_{t}=\rho C_{\mu} \frac{k^{2}}{\epsilon}$

$C_{\mu}=0.009$ (Model constant for Turbulent viscosity)

$C_{1 \varepsilon}=1.44$ (Model constant for transport equation)

$C_{2 \varepsilon}=1.92$ (Model constant for transport equation)

$\sigma_{k}=1.0$ (Turbulent kinetic energy Prandtl number)

$\sigma_{\varepsilon}=1.3$ (Turbulent dissipation rate Prandtl number)

But $\mathrm{k}-\varepsilon$ model lags in a variety of important cases such as unconfined flows, curved boundary layers and in cases of large adverse pressure gradients.

These deficiencies were rectified by the realizable $\mathrm{k}-\varepsilon$ model by incorporating:

- $\quad$ eddy-viscosity formula involving variable $C_{\mu}$

- model equation for dissipation based on the dynamic equation of the mean square velocity fluctuation.

This refined model helps in achieving an accurate integral value whose accuracy ranges from $2-5 \%$ from the actual. Also the model is stable and converges even quicker [14].

\section{PRE PROCESSING}

\subsection{Vehicle Geometry Modeling}

The vehicle once being selected was modeled on the CAD software by the dimensions of the model that was obtained. The side profile of the vehicle was drawn and then extruded to the width of the vehicle. The wheel arches were not constructed along with the tyres, as flow around the tyres and inside the wheel well was outside the scope of this project. Once completed the model was imported into ANSYS workbench where it was renamed as Honda City for easy identification in the history chart. Fig.3. shows an isometric model of the same within the Design Modeler. After meshing it was found that the number of elements was too large (approx.8 million) for my workstation to handle smoothly without lagging. So a 1:4 scale model was then designed and imported into ANSYS which led to a drop in the number of elements but the aerodynamic forces acting on it would be the same so results won't vary from full sized model.

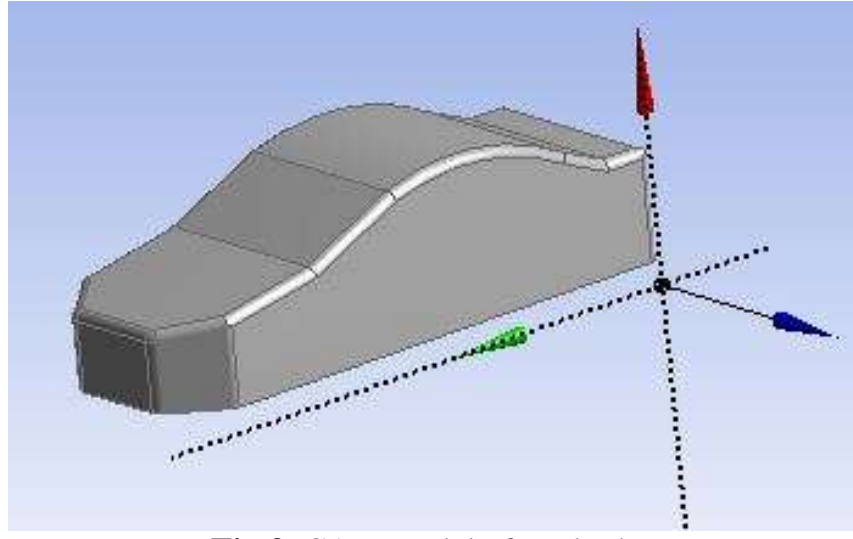

Fig.3. CAD Model of car body

\subsection{Diffuser Design}

The airflow exiting the diffuser has to match the outside higher air pressure as it results in pressure-induced drag which is again undesirable. So, smoothing out the turbulent air exiting towards the rear of the car and matching the outside air at high pressure while minimizing the drag is very critical. As the diffuser has minimum drag penalties than other aerodynamic devices, it has turned out to be most efficient for downforce generation. I designed a simple diffuser whose height was kept constant to ensure that the pressure variation does not occur and changed the angle of diffuser. Also, as many designs suggested a need for vanes in the diffuser to ensure laminar flow of the air going through it so there are four vanes along the length of the diffuser. These are equidistant from each other and are symmetrical about the centre axis of the vehicle Fig4. Along the sides due to the presence of wheels, a space was left for the tyres to ensure that the results are as close to actual as possible. Since the height of the diffuser was fixed at $60.5 \mathrm{~mm}$ (scale model), the length of the diffuser varied till half the length of the vehicle.

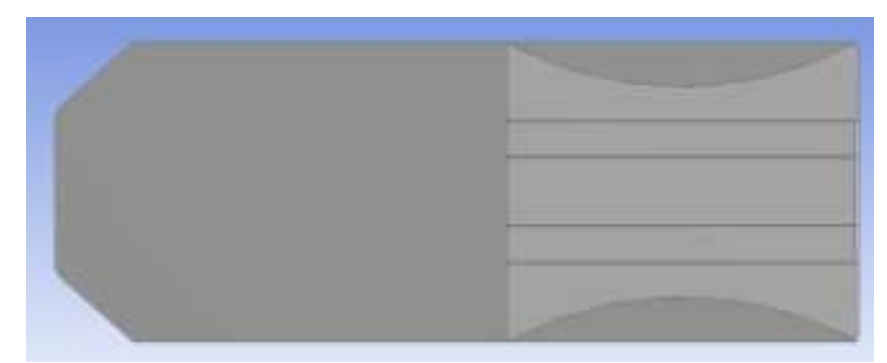

Fig 4.Diffuser model designed for the car body.

\subsection{Creating Fluid Enclosure}

The vehicle needs to be put in a virtual canopy which has properties of air so as to study its aerodynamic resistances. So this was done by creating an enclosure around the vehicle body and then subtracting the vehicle body from it to retain the "air" around the car sans the body. The air domain created was 3 car lengths both ahead and from the top of the car, and at the rear where the turbulence is to be studied, it was kept at 5 car lengths. To simply the simulation time and cost, the vehicle was taken to be symmetrical laterally. 


\subsection{Mesh Generation}

During mesh generation, the domains where the results were to be studied thoroughly were meshed with smaller sized elements which would grow in size as they move away from the car where accuracy was not required as shown in Fig5. This helps to reduce computational time as well.

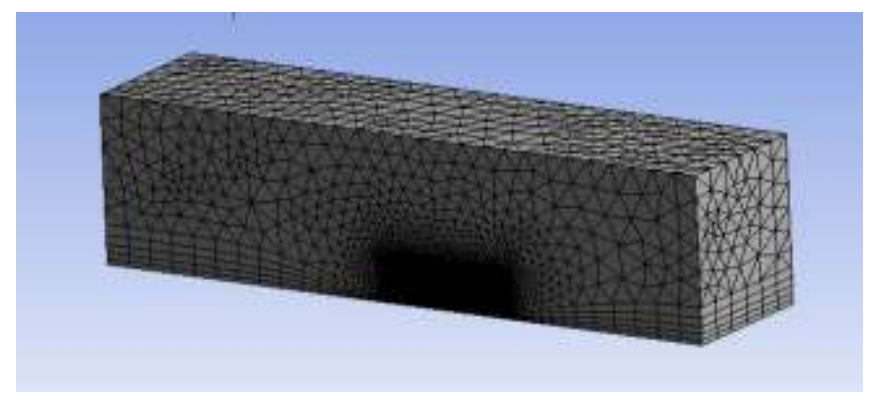

The immediate vicinity of the car (Fig6.) was surrounded by fine mesh also beneath the car body to understand the flow of air below the vehicle. Also from about halfway of the car the mesh was further refined in the wake region towards the rear. This helps to accurately predict the flow separation taking place at the roof to the vortex generation at the rear and its convergence with the calm air. Also boundary layer separation being of massive importance, five layers of inflation was added around the vehicle surface and the road to resolve the boundary layer smoothly. The total number of elements came out to be 1.781 million elements. The entire mesh developed was then checked for its skewness which came out to be 0.845 as its minimum value. ANSYS does not solve mesh with skewness parameter more than 0.95 [15], and the created mesh turned out to be a good mesh.

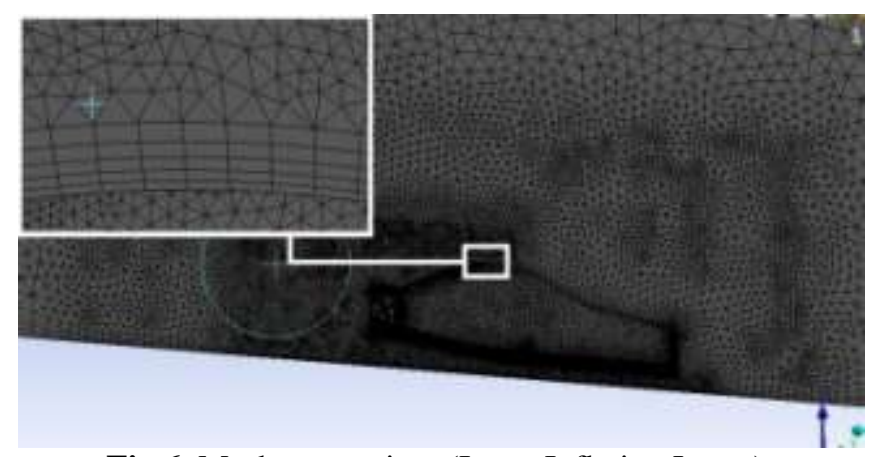

Fig 6. Mesh generation. (Inset: Inflation Layer)

\subsection{Boundary Conditions}

The enclosure was then named on all of it faces in which the inlet face was "velocity-inlet". The outlet face was the "pressure-outlet". Based on this the solver automatically assumes which is which and prepares a model for constant pressure output i.e. atmospheric pressure with a certain inlet air velocity to be given by the user. The velocities for the simulations were 30,35 and $45 \mathrm{~m} / \mathrm{s}$. Based on the other names given to the enclosure surfaces, it identifies them as wall surfaces and the symmetry surfaces at the top and side was then considered as "no-slip" condition by the solver.

\section{SOLVER}

In this analysis a pressure based steady state solver was used. The various other changes in the Fluent window are listed as below:

- Pressure Based Steady State Solver

- Realizable k- $\varepsilon$ model with non-equilibrium wall functions

- Air velocity at inlet: $40 \mathrm{~m} / \mathrm{s}$

- Reference Area to determine drag and lift coefficientsFrontal Area: $0.068818 \mathrm{~m}^{2}$

Then the first step was to perform hybrid initialization. Providing an initial data range that is very close to the final solution for steady-state simulations means that the solver has to do less work to reach the converged result. Therefore, this reduces simulation time and the cost.

The final solution was obtained in a two-step process with progressive iteration. In both the stages, the accuracy of the solver was increased by employing higher order equations. In the first stage the solution was prevented from diverging by employing first order equations. The Coupled solver was used to complete the simulations and once sufficient convergence was reached at the scaled residuals, the second order equations were carried out by solver. And it was allowed to continue till the fifth decimal point of the drag coefficient came to a point where it was almost the same number.

Table1:Model Analysis Methodology applied

\begin{tabular}{|l|l|l|}
\hline Stage & I & II \\
\hline Scaled Residuals Convergence & $10 \mathrm{e}^{-4}$ & $10 \mathrm{e}^{-4}$ \\
\hline Iteration number & 100 & 500 \\
\hline Order of Equation Solved & First Order & Second Order \\
\hline Turbulence Viscosity & 0.8 & 0.95 \\
\hline Solver & Coupled & Coupled \\
\hline Time Taken & 1.5 hours & $8-8.5$ hours \\
\hline
\end{tabular}

\subsection{Solver Accuracy}

\section{Test Model}

The simulations were calibrated by simulating an Ahmed Body using the same solver methodology and its result was compared with theoretically found results. The drag coefficient originally calculated for Ahmed Body comes out to 0.285 , whereas the simulations carried out by the simulations gave out drag coefficient as 0.282 . A difference in thousandths is enough to justify the accuracy of our solver model and further simulations for the models can be carried out.

\section{RESULTS}

The drag coefficient obtained for the base model was. The analysis of the wheel well, radiators, rotating tyres are renowned to impart lot of induced drag upon the vehicle and contribute upto $25-28 \%$ of the total drag. Adding these drag penalties to the obtained values of $\mathrm{Cd}$, we get its new value 
in the range of 0.302-0.32736 which seems in the ballpark of the vehicles similar to this. So we are assured that our simulations are correct and hence plot the results for the same.

The total pressure, turbulence kinetic energy, and velocity contours are shown below for base model at $40 \mathrm{~m} / \mathrm{s}$ wind velocity without the diffuser installed

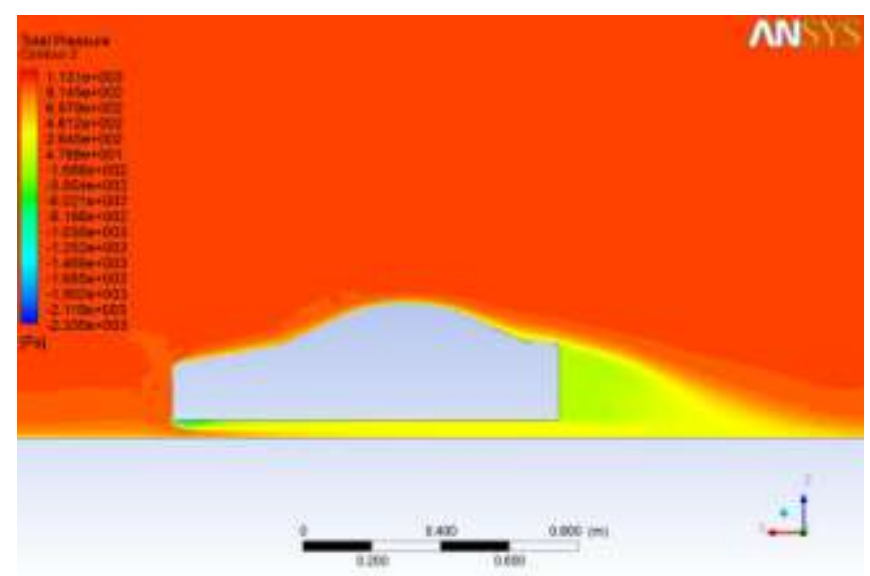

Fig 7. Total Pressure

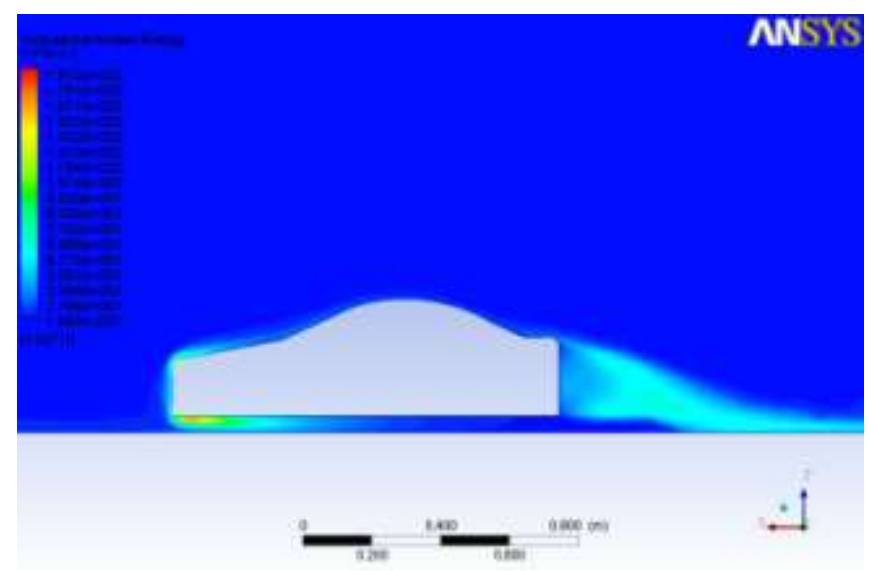

Fig 8. Turbulence kinetic energy

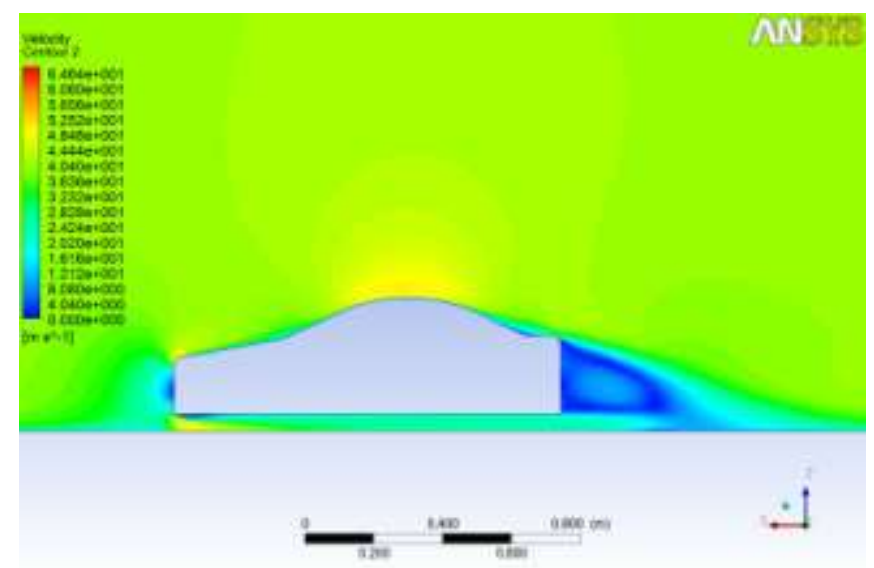

Fig 9.Velocity Contour

Now after adding diffuser of $8^{\circ}$ we got the best results as shown in the following contours:

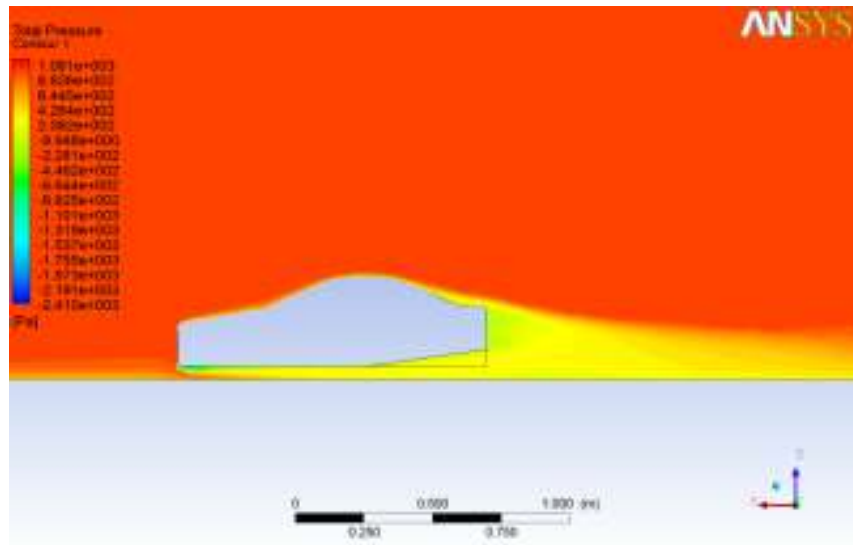

Fig 10.Total Pressure

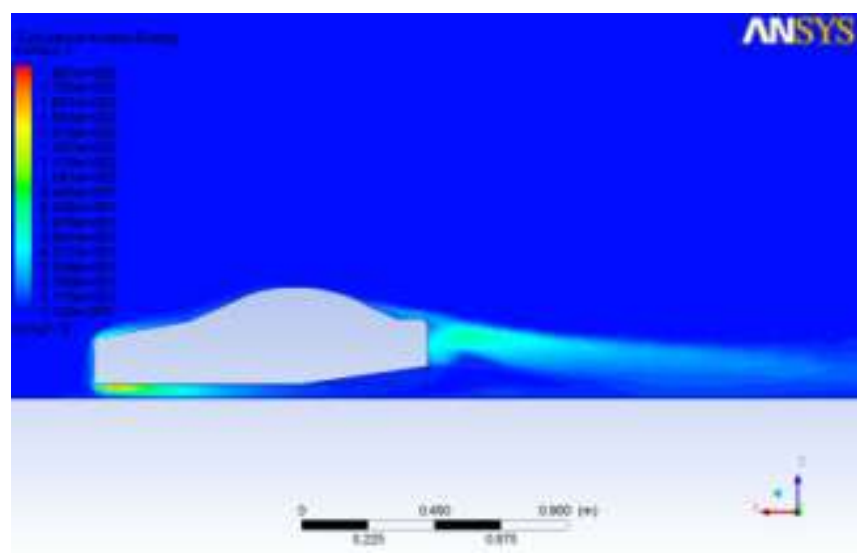

Fig 11.Turbulence Kinetic Enery

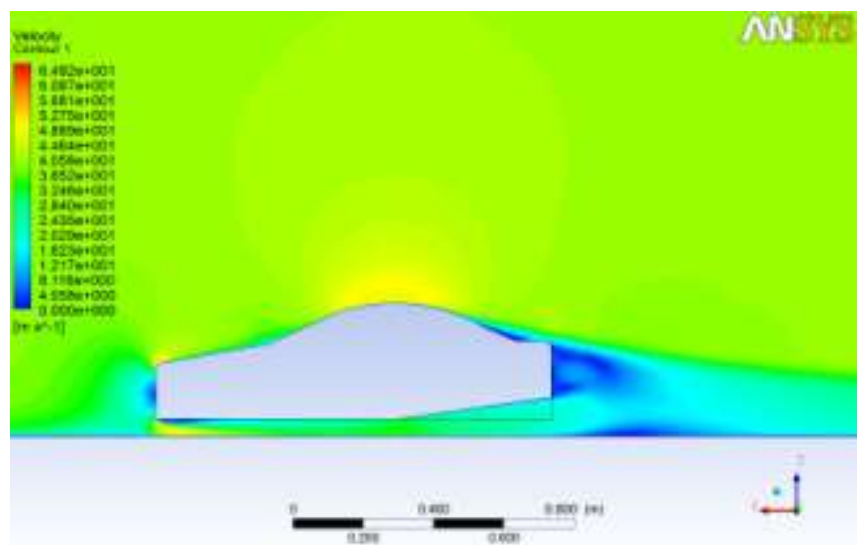

Fig 12. Velocity Contour

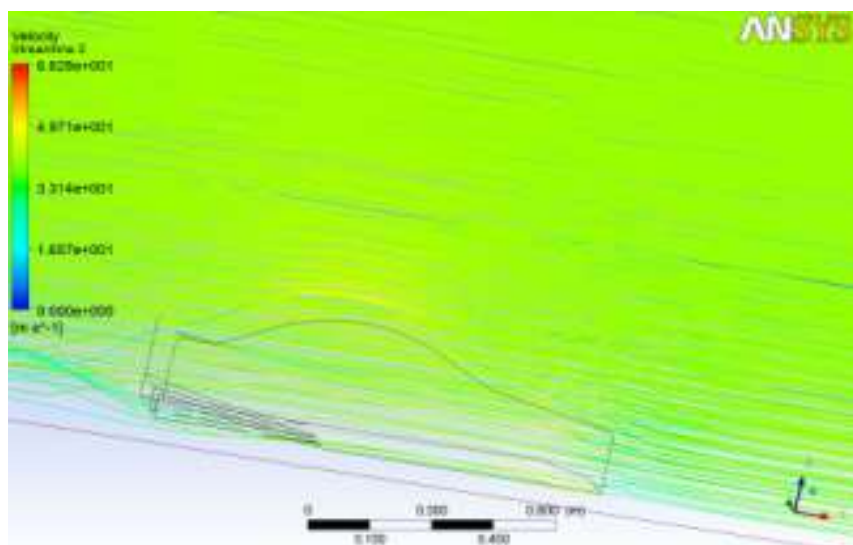

Fig 13.Velocity Streamlines around car body 
The various results obtained for the diffuser angles and wind velocities are as shown below:

Table 2: Drag Coefficient values obtained from the simulations at various diffuser angles and wind velocities

\begin{tabular}{|l|l|l|l|}
\hline & $30 \mathrm{~m} / \mathrm{s}$ & $35 \mathrm{~m} / \mathrm{s}$ & $40 \mathrm{~m} / \mathrm{s}$ \\
\hline Base Model & 0.23665 & 0.23516 & 0.2339 \\
\hline Diffuser Angle $5^{\circ}$ & 0.243489 & 0.242243 & 0.2412 \\
\hline${\text { Diffuser Angle } 7^{\circ}}^{\circ}$ & 0.2335 & 0.231767 & 0.23116 \\
\hline Diffuser Angle $8^{\circ}$ & 0.25575 & 0.25491 & 0.25425 \\
\hline Diffuser Angle $10^{\circ}$ & 0.25567 & 0.254613 & 0.25398 \\
\hline
\end{tabular}

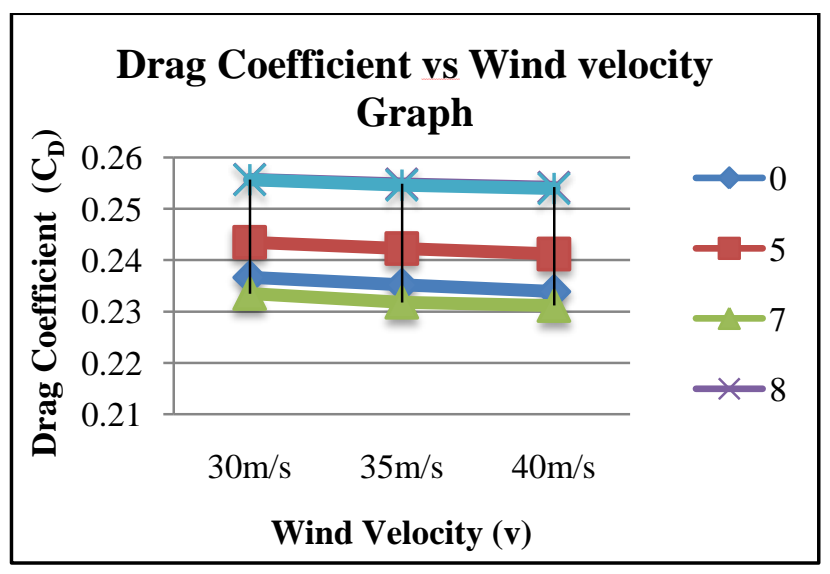

Diffusers are understood to not help in improving the drag coefficient but they also don't alter it in any major way as can be seen above. The diffuser at angles 8 and 10 cause the maximum increase in the $C_{D}$ whereas at 7 deg the diffuser actually reduces the drag very slightly to the tune of 1.3-1.4 $\%$.

Table 3: Lift Coefficient values obtained from the simulations at various diffuser angles and wind velocities

\begin{tabular}{|l|l|l|l|}
\hline & $30 \mathrm{~m} / \mathrm{s}$ & $35 \mathrm{~m} / \mathrm{s}$ & $40 \mathrm{~m} / \mathrm{s}$ \\
\hline Base Model & 0.157452 & 0.156729 & 0.156193 \\
\hline Diffuser Angle $5^{\circ}$ & -0.187599 & -0.19421 & -0.2122 \\
\hline Diffuser Angle $7^{\circ}$ & -0.252015 & -0.264109 & -0.27393 \\
\hline Diffuser Angle $8^{\circ}$ & -0.286967 & -0.296707 & -0.305947 \\
\hline Diffuser Angle $10^{\circ}$ & -0.288861 & -0.297573 & -0.305777 \\
\hline
\end{tabular}

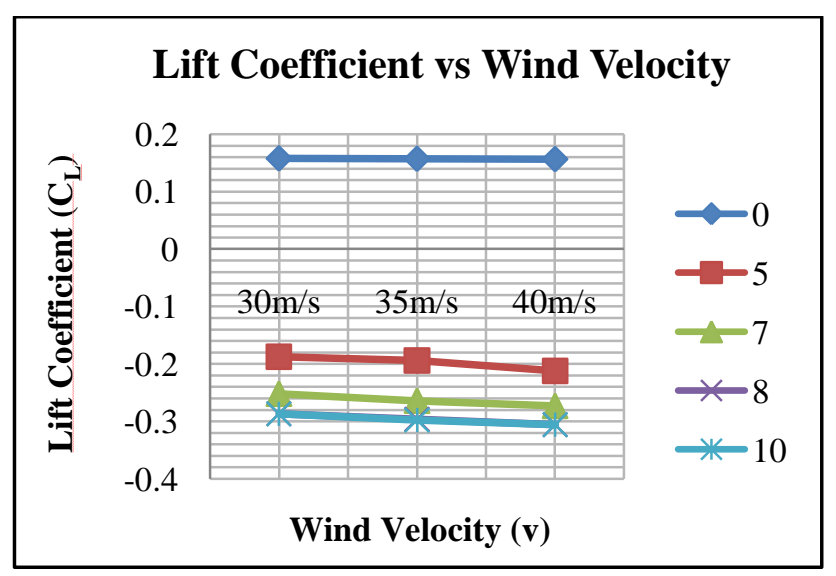

As can be seen from the graph, the introduction of the diffuser has created massive enhancements in the lift coefficient. The base model has lift forces acting on it and has $C_{L}$ ranging from $0.157-0.156$. And it has a positive sign which indicates the lift forces acting on the car body. But when the diffuser is introduced, the lift coefficient changes to negative due to the formation of downforce by it. The most effective angle of diffuser hence found was $8^{\circ}$. And the percentage improvement in lift forces when calculated comes out to be about $296 \%$. So it can be concluded that the diffuser design has been very successful and barring few design changes it can be brought into production.

Along with this another interesting aspect to look into would be the weight reduction because of the presence of diffuser. As the length of the diffuser varies according to the angle of it, it shaves off a lot of bodywork from beneath which can result in increased performance because of lighter weight. Checking the official Honda website I found out that the kerb weight for the top end VX model was $1065 \mathrm{~kg}$. Then I proceeded to my base model that I designed on Solidworks and obtained the volume for the same which came out to around $1.1689 * \mathrm{e}^{8} \mathrm{~mm}^{3}$. So I calculated the density for the model which turned out to be $911.13 \mathrm{~kg} / \mathrm{m}^{3}$. Using this density volume I proceeded to calculate the weight of the vehicle when incorporated with the diffuser which is shown as below:

Table 4: Volume and weight of every model analysed

\begin{tabular}{|l|l|l|}
\hline Model Type & Volume $\left(\mathrm{mm}^{3}\right)$ & Weight $(\mathrm{kg})$ \\
\hline Base Model & $1.1689 * \mathrm{e}^{8}$ & 1065 \\
\hline Diffuser angle $5^{\circ}$ & $1.1013 * \mathrm{e}^{8}$ & 1003.43 \\
\hline Diffuser angle $7^{\circ}$ & $1.1204 * \mathrm{e}^{8}$ & 1020.83 \\
\hline Diffuser angle $8^{\circ}$ & $1.1273 * \mathrm{e}^{8}$ & 1027.11 \\
\hline Diffuser angle $10^{\circ}$ & $1.1353 * \mathrm{e}^{8}$ & 1034 \\
\hline
\end{tabular}

As can be seen from above, the diffuser has led to reduction in the weight of the vehicle. In the case of diffuser angle of $5^{\mathrm{o}}$ it gives most reduction because of its increased length. The reduction in weight comes to around $5.78 \%$ which is considerable.

\section{CONCLUSION}

$>$ Diffuser design has shown that it increases the drag coefficient of the vehicle but it is very low in value and won't affect the performance of the vehicle.

$>$ Diffuser design of angle $7^{\circ}$ has been found to give an improvement in drag performance but that is also on the lower side to the region of $1.3-1.4 \%$.

$>$ Diffuser design of angle $8^{\circ}$ was found to give most efficient downforce. At $40 \mathrm{~m} / \mathrm{s}$ it gives maximum downforce of -0.305947 . The improvement in the result obtained from base model comes to $296 \%$ because it has converted lift force into downforce.

$>$ All the graphs denote that the drag increases with increase in speed because drag force and velocity are directly relative to each other. 
$>$ The downforce of the vehicle increases with an increase in speed due to higher pressure acting on the vehicle.

$>$ The diffuser design has led to reduction in weight by upto $5.78 \%$ which is pretty impressive.

$>$ The downforce generation by diffusers of angles $8^{\circ}$ and $10^{\circ}$ was very identical and hence it can be safely assumed that the for angles greater than $10^{\circ}$ the flow would separate and the diffuser might fail.

\section{FUTURE SCOPE}

$>$ Study of flow around wheel arches and over rotating wheels was beyond the feasibility of my workstation, so further enlightment on that is desirable.

$>$ Vortex generators, spoilers, gurney flaps and plenty other aerodynamic devices are present which can be designed for any particular vehicle and its study can be presented.

$>$ Diffusers usually work in optimum capacity when the car has a spoiler at its rear. So a combination of these two and their effects on the car can be studied.

$>$ Also my work needs to be calibrated with wind tunnel tests, so as to ascertain the accuracy of my simulations.

$>$ Another scope can be study of different designs of diffusers along with materials for the same to be used.

\section{REFERENCES}

[1]. National Highways Authority of India(2010). "Indian Road Network" Ministry ofRoad Transport \& Highwayshttp://www.nhai.org/roadnetwork.html

[2]. National Crime Records Bureau (2009).“Accidental Deaths \& Suicides in India,2009,"NCRB, Ministry of Home Affairs, 2010.

[3]. Analysis of In-Depth Crash Data on Indian National Highways and Impact of Road Design on Crashes and Injury Severity, J. Padmanaban, R. Rajaraman, G. Stadter, S. Narayan, B. Ramesh, JP Research, Inc.

[4]. NHTSA, UK 2005.

[5]. J.D.Anderson Jr., Computational Fluid Dynamics: The Basics with Applications, McGraw Hill Publication, 1995.

[6]. Ground Effect Aerodynamics of Race Cars, X. Zhang, W. Toet, J. Zerihan

[7]. www.rapid-racer.com

[8]. Asif A and Murtaza MA, "CFD Analysis of Car Body Aerodynamics including Effect of Passive Flow Devices", International Journal of Research in Engineering and Technology, Vol 5, Issue 3, pp 141-144, March 2016.

[9]. Race car aerodynamics, GregorSeljak, April8,2008

[10]. Cooper, K. R., Bertenyi, T., Dutil, G., Syms, J., and Sovran, G., 1998, "The Aerodynamic Performance of Automotive Underbody Diffusers," Technical Report SAE Paper 98-0030, Society of Automotive Engineers, Warrendale, PA, USA.

[11]. Cooper, K. R., Sovran, G., and Syms, J., 2000, "Selecting Automotive Diffusers to Maximise Underbody Downforce," SAE Paper 2000-01-0354.

[12]. Accelerating CFD Solutions, Mark Keating, Principal Engineer, ANSYS Inc.

[13]. Launder and Spalding,"The study of the k- $\varepsilon$ model", 1974.
[14]. Aerodynamic Analysis of a Car Model using FluentAnsys 14.5, A.Parab, A. Sakarwala, B. Paste, V. Patil, IJRMEE , Nov-2014.

[15]. Best practice guidelines for handlingAutomotive External Aerodynamics with FLUENT, Version 1.2(Feb 9th 2005), Marco Lanfrit, Fluent Deutschland GmbH. 